\title{
LOD-BOR-FDTD Algorithm for Efficient Analysis of Circularly Symmetric Structures
}

\author{
Jun Shibayama, Member, IEEE, Bungo Murakami, Junji Yamauchi, Senior Member, IEEE, and \\ Hisamatsu Nakano, Fellow, IEEE
}

\begin{abstract}
The unconditionally stable locally 1-D (LOD) scheme is used to develop an efficient implicit body-of-revolution (BOR) finite-difference time-domain (FDTD) method. In the LOD-BORFDTD, the number of arithmetic operations of the resultant finite-difference equations is significantly reduced, when compared with the alternating-direction implicit (ADI) BOR-FDTD. Numerical results of circular cavity resonators reveal that the LOD-BORFDTD provides resonance frequencies identical to the ADI counterparts, with the computational time being reduced to $70 \%$.
\end{abstract}

Index Terms-Body of revolution (BOR), locally 1-D finitedifference time-domain method (LOD-FDTD), rotationally symmetric geometry, unconditional stability.

\section{INTRODUCTION}

$\mathbf{T}$ HE body-of-revolution finite-difference time-domain (BOR-FDTD) method [1]-[3] is an effective means for treating electromagnetic problems of circularly symmetric structures. Note, however, that as in the conventional FDTD a time step size of the BOR-FDTD is limited by the Courant-Friedrich-Levy (CFL) condition. This limitation has been removed [4] using the alternating-direction implicit (ADI) scheme [5], [6]. Recently, the weakly conditionally stable scheme [7] has been applied to the BOR-FDTD [8], in which the time step size is subject to only single space discretization.

On the other hand, the locally 1-D (LOD) scheme has been introduced to the unconditionally stable implicit FDTD formulation [9]-[11]. The LOD-FDTD provides a quite simple algorithm compared with the ADI algorithm. Strictly speaking, the LOD formulation is a simple type of split-step approach [12] and is first-order accurate in time [11], whereas the ADI formulation is second-order accurate in time. Nevertheless, the numerical results obtained from the LOD-FDTD have been found to be comparable to the ADI counterparts for not only the 2-D [9], [11], [13] but also 3-D problems [14]-[16]. Therefore, the LOD-FDTD could be a practical alternative for an efficient implicit FDTD. This letter is devoted to the application of the LOD scheme to the BOR-FDTD for efficient implicit calculations of circularly symmetric structures. The usefulness of the LOD-BOR-FDTD is investigated through the analysis of circular cavity resonators with and without a dielectric disc, along with the explicit BOR-FDTD and ADI-BOR-FDTD.

Manuscript received September 03, 2008; revised October 22, 2008. Current version published February 11, 2009. This work was supported in part by MEXT, Grant-in-Aid for Scientific Research (c) (19560355).

The authors are with the Faculty of Engineering, Hosei University, Tokyo 184-8584, Japan (e-mail: shiba@hosei.ac.jp).

Digital Object Identifier 10.1109/LMWC.2008.2011302

\section{FORMULATION}

In the circularly symmetric structures, the fields can be expanded in a Fourier series of sines and cosines as [1]

$$
\begin{aligned}
& E=\sum_{m=0}^{\infty}\left(E_{\text {even }, m} \cos m \phi+E_{\text {odd }, m} \sin m \phi\right) \\
& H=\sum_{m=0}^{\infty}\left(H_{\text {even }, m} \cos m \phi+H_{\text {odd }, m} \sin m \phi\right)
\end{aligned}
$$

where $m$ is the mode number. Substituting (1) into Maxwell's equations in cylindrical coordinates, we obtain the following equation in the matrix form:

$$
\frac{\partial \boldsymbol{u}}{\partial t}=([A]+[B]) \boldsymbol{u}
$$

where $\boldsymbol{u}=\left[E_{\rho}, E_{\phi}, E_{z}, H_{\rho}, H_{\phi}, H_{z}\right]^{T}$ and

$$
\begin{aligned}
& {[A]=\left[\begin{array}{cccccc}
0 & 0 & 0 & 0 & -\frac{\partial}{\epsilon \partial z} & 0 \\
0 & 0 & 0 & 0 & 0 & -\frac{\partial}{\epsilon \partial \rho} \\
0 & 0 & 0 & -\frac{m}{\epsilon \rho} & 0 & 0 \\
0 & 0 & \frac{m}{\mu \rho} & 0 & 0 & 0 \\
-\frac{\partial}{\mu \partial z} & 0 & 0 & 0 & 0 & 0 \\
0 & -\frac{\partial(\rho)}{\mu \rho \partial \rho} & 0 & 0 & 0 & 0
\end{array}\right]} \\
& {[B]=\left[\begin{array}{cccccc}
0 & 0 & 0 & 0 & 0 & \frac{m}{\epsilon \rho} \\
0 & 0 & 0 & \frac{\partial}{\epsilon \partial z} & 0 & 0 \\
0 & 0 & 0 & 0 & \frac{\partial(\rho)}{\epsilon \rho \partial \rho} & 0 \\
0 & \frac{\partial}{\mu \partial z} & 0 & 0 & 0 & 0 \\
0 & 0 & \frac{\partial}{\mu \partial \rho} & 0 & 0 & 0 \\
-\frac{m}{\mu \rho} & 0 & 0 & 0 & 0 & 0
\end{array}\right]}
\end{aligned}
$$

in which $\epsilon$ and $\mu$ represent permittivity and permeability, respectively. Applying the Crank-Nicolson scheme to (2) gives

$$
\boldsymbol{u}^{n+1}=\frac{[I]+\frac{\Delta t}{2}([A]+[B])}{[I]-\frac{\Delta t}{2}([A]+[B])} \boldsymbol{u}^{n}
$$

where $I$ denotes the unit matrix. We discretize (3) using the LOD scheme, so that

$$
\boldsymbol{u}^{n+1 / 2}=\frac{[I]+\frac{\Delta t}{2}[B]}{[I]-\frac{\Delta t}{2}[B]} \boldsymbol{u}^{n}
$$

for the first step and

$$
\boldsymbol{u}^{n+1}=\frac{[I]+\frac{\Delta t}{2}[A]}{[I]-\frac{\Delta t}{2}[A]} \boldsymbol{u}^{n+1 / 2}
$$

for the second step. 
TABLE I

NUMBER OF ARITHMETIC OPERATIONS

\begin{tabular}{|c|c|c|c|c|c|c|}
\hline \multirow{2}{*}{ Method } & \multicolumn{2}{|c|}{ Implicit } & \multicolumn{2}{c|}{ Explicit } & \multicolumn{2}{c|}{ Total } \\
\cline { 2 - 7 } & M/D & A/S & M/D & A/S & M/D & A/S \\
\hline ADI & 12 & 20 & 13 & 20 & 25 & 40 \\
\hline LOD & 11 & 16 & 5 & 16 & 16 & 32 \\
\hline
\end{tabular}

For simplicity, we will examine the case for $m=0$, so that the LOD-BOR-FDTD equations are derived as

$$
\begin{aligned}
& E_{\rho}^{n+1 / 2}=E_{\rho}^{n} \\
& E_{\phi}^{n+1 / 2}-\frac{\Delta t}{2 \epsilon} \frac{\partial H_{\rho}^{n+1 / 2}}{\partial z}=E_{\phi}^{n}+\frac{\Delta t}{2 \epsilon} \frac{\partial H_{\rho}^{n}}{\partial z} \\
& E_{z}^{n+1 / 2}-\frac{\Delta t}{2 \epsilon \rho} \frac{\partial\left(\rho H_{\phi}^{n+1 / 2}\right)}{\partial \rho}=E_{z}^{n}+\frac{\Delta t}{2 \epsilon \rho} \frac{\partial\left(\rho H_{\phi}^{n}\right)}{\partial \rho} \\
& H_{\rho}^{n+1 / 2}-\frac{\Delta t}{2 \mu} \frac{\partial E_{\phi}^{n+1 / 2}}{\partial z}=H_{\rho}^{n}+\frac{\Delta t}{2 \mu} \frac{\partial E_{\phi}^{n}}{\partial z} \\
& H_{\phi}^{n+1 / 2}-\frac{\Delta t}{2 \mu} \frac{\partial E_{z}^{n+1 / 2}}{\partial \rho}=H_{\phi}^{n}+\frac{\Delta t}{2 \mu} \frac{\partial E_{z}^{n}}{\partial \rho} \\
& H_{z}^{n+1 / 2}=H_{z}^{n}
\end{aligned}
$$

for the first step and

$E_{\rho}^{n+1}+\frac{\Delta t}{2 \epsilon} \frac{\partial H_{\phi}^{n+1}}{\partial z}=E_{\rho}^{n+1 / 2}-\frac{\Delta t}{2 \epsilon} \frac{\partial H_{\phi}^{n+1 / 2}}{\partial z}$

$E_{\phi}^{n+1}+\frac{\Delta t}{2 \epsilon} \frac{\partial H_{z}^{n+1}}{\partial \rho}=E_{\phi}^{n+1 / 2}-\frac{\Delta t}{2 \epsilon} \frac{\partial H_{z}^{n+1 / 2}}{\partial \rho}$

$E_{z}^{n+1}=E_{z}^{n+1 / 2}$

$H_{\rho}^{n+1}=H_{\rho}^{n+1 / 2}$

$H_{\phi}^{n+1}+\frac{\Delta t}{2 \mu} \frac{\partial E_{\rho}^{n+1}}{\partial z}=H_{\phi}^{n+1 / 2}-\frac{\Delta t}{2 \mu} \frac{\partial E_{\rho}^{n+1 / 2}}{\partial z}$

$H_{z}^{n+1}+\frac{\Delta t}{2 \mu \rho} \frac{\partial\left(\rho E_{\phi}^{n+1}\right)}{\partial \rho}=H_{z}^{n+1 / 2}-\frac{\Delta t}{2 \mu \rho} \frac{\partial\left(\rho E_{\phi}^{n+1 / 2}\right)}{\partial \rho}$

for the second step. For $m=0$, we must pay attention to handling the singular point at $\rho=0$. Instead of (5c), we adopt the following equation in accordance with LOD implementation:

$$
E_{z}^{n+1 / 2}=E_{z}^{n}+\frac{4 \Delta t}{\epsilon \Delta \rho} H_{\phi}^{n+1 / 2} .
$$

Now, we solve the above equations. For the TE mode in which the $E_{\phi}, H_{\rho}$ and $H_{z}$ fields are calculated, we substitute (5d) into (5b) and implicitly solve the resultant tridiagonal system of linear equations for $E_{\phi}^{n+1 / 2}$, and then explicitly solve (5d) for $H_{\rho}^{n+1 / 2}$. Next, we substitute (6f) into (6b) and implicitly solve the resultant tridiagonal system for $E_{\phi}^{n+1}$, and then explicitly solve (6f) for $H_{z}^{n+1}$. The equations for the TM mode can similarly be solved for the $H_{\phi}, E_{\rho}$ and $E_{z}$ fields.

Here, we count the number of arithmetic operations of the right-hand side of the resultant finite-difference equations for

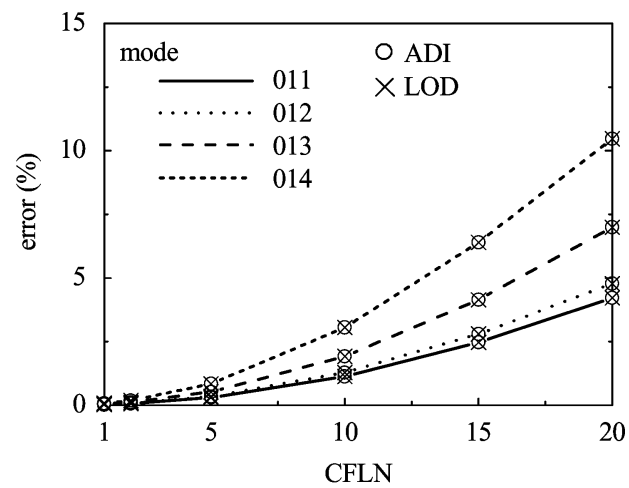

(a)

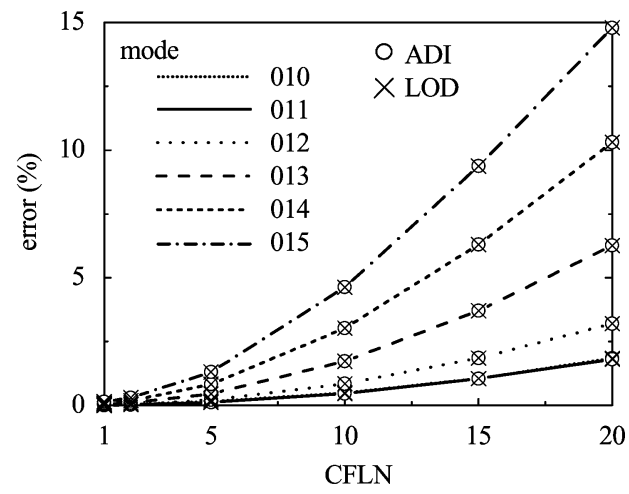

(b)

Fig. 1. Error of the resonance frequency versus CFLN. In (b), the errors of the $\mathrm{TM}_{010}$ mode are almost superimposed onto those of the $\mathrm{TM}_{011}$ mode. (a) $\mathrm{TE}$ mode, (b) TM mode.

$m=0$, in comparison with the ADI counterparts. Table I summarizes the results, in which M/D and A/S indicate multiplication/division and addition/subtraction operations, respectively. It is clear that the number for the LOD case is reduced when compared with the ADI case. A reduction becomes significant for $m \neq 0: \mathrm{M} / \mathrm{D}$ and $\mathrm{A} / \mathrm{S}$ are, respectively, 22 and 38 in total for the LOD, while they are 40 and 56 for the ADI. As a result, the algorithm can simply be implemented with the LOD scheme.

\section{NUMERICAL RESULTS}

To assess the LOD-BOR-FDTD in terms of accuracy and efficiency, we analyze the two circular cavity resonators frequently used as a benchmark [3], [4], [17]. The first example is the circular cavity formed by closing the two ends of the waveguide with plates [18], [19]. The radius and height of the cavity are $3.995 \mathrm{~cm}$ and $7.910 \mathrm{~cm}$, respectively. The sampling widths are $\Delta \rho=0.799 \mathrm{~mm}$ and $\Delta z=0.791 \mathrm{~mm}$. We follow the calculation parameters used in [4], in which a Gaussian pulse with frequency content up to $6 \mathrm{GHz}$ is excited.

Figs. 1(a) and (b) show the errors of the resonance frequencies for the TE and TM modes, respectively, versus time step size CFLN defined by $\Delta t / \Delta t_{\mathrm{CFL}}$ where $\Delta t_{\mathrm{CFL}}=1.3183 \mathrm{ps}$ is determined by the CFL condition. The theoretical values of the resonance frequencies are available from [18], [19] (our careful calculation reveals that the resonance frequency of the $\mathrm{TE}_{011}$ mode is $4.953 \mathrm{GHz}$, whereas it is denoted as $4.950 \mathrm{GHz}$ in [4], [17]). The higher-order modes are also investigated. It is found 


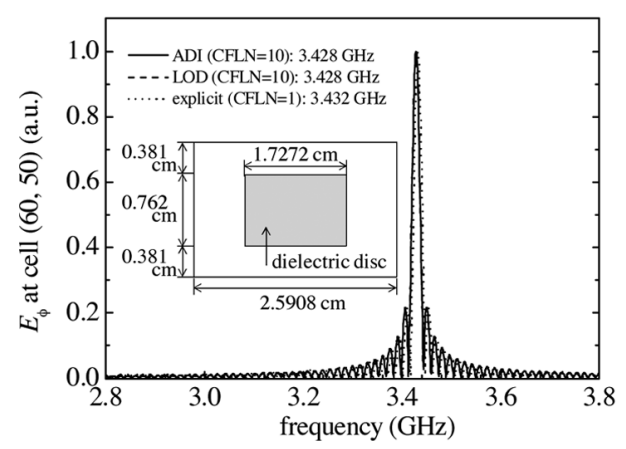

Fig. 2. $E_{\phi}$ versus frequency. The inset shows a cavity resonator to be studied. The LOD result is almost superimposed onto the ADI result.

that the results of the LOD-BOR-FDTD are in agreement with those of the ADI-BOR-FDTD, in which the error increases with CFLN due to the numerical dispersion. In addition, as the mode becomes higher the error increases, which is caused by a steeper variation of the mode shape. Although not illustrated, the error of the explicit BOR-FDTD for CFLN $=1$, relative to the theoretical value, is always less than $0.1 \%$ in this example. We here mention the efficiency of the LOD-BOR-FDTD. The computational times, e.g., for $\mathrm{CFLN}=10$, are $12.3 \mathrm{~s}$ and $17.5 \mathrm{~s}$ for the LOD- and ADI-BOR-FDTDs, respectively, while the time is $42.7 \mathrm{~s}$ for the explicit BOR-FDTD, where a PC with a Pentium 4 processor $(3.8 \mathrm{GHz})$ is utilized. This indicates the time reductions to $70 \%$ and $30 \%$ of the ADI and explicit counterparts, respectively.

The second example is the circular cavity with the dielectric disc $\left(\epsilon_{r}=35.74\right)$ being centered in the cavity [4], [17] (the inset in Fig. 2). The sampling widths are $\Delta \rho=0.17272 \mathrm{~mm}$ and $\Delta z=0.1524 \mathrm{~mm}$. Fig. 2 shows the strength of $E_{\phi}$ versus frequency, in which CFLN $=10$ is used for the LOD- and ADI-BOR-FDTDs. Again, we can find excellent agreement between the LOD and ADI results, yielding the identical resonance frequency. Although these results slightly deviate from the explicit result (dotted line), the difference in resonance frequency is only $0.12 \%$. We also obtain the same efficiency improvement for the LOD calculations as that mentioned in the first example.

\section{CONCLUSION}

The efficient implicit BOR-FDTD has been formulated on the basis of the unconditionally stable LOD scheme. The LODBOR-FDTD offers the quite simple algorithm with a subsequent reduction in the computational time, maintaining numerical results identical to the ADI counterparts. The LOD formulation can also be applied to the cylindrical coordinate system [17]. For open region problems, efficient absorbing boundary conditions such as perfectly matched layers [20]-[24] are required, the application of which is left for a future study. Further improvement in efficiency may be anticipated if the LOD is implemented in its fundamental scheme [25].

\section{REFERENCES}

[1] A. Taflove and S. C. Hagness, Computational Electrodynamics: The Finite-Difference Time-Domain Method,3rd ed. Norwood, MA: Artech House, 2005, ch. 12.
[2] D. B. Davidson and R. W. Ziolkowski, "Body-of-revolution finite-difference time-domain modeling of space-time focusing by a three-dimensional lens," J. Opt. Soc. Amer. A, vol. 11, no. 4, pp. 1471-1490, Apr. 1994.

[3] Y. Chen, R. Mittra, and P. Harms, "Finite-difference time-domain algorithm for solving Maxwell's equations in rotationally symmetric geometries," IEEE Trans. Microw. Theory Tech., vol. 44, no. 6, pp. 832-839, Jun. 1996.

[4] H.-L. Chen, B. Chen, Y. Yi, and D.-G. Fang, "Unconditionally stable ADI-BOR-FDTD algorithm for the analysis of rotationally symmetric geometries," IEEE Microw. Wireless Compon. Lett., vol. 17, no. 4, pp. 304-306, Apr. 2007.

[5] T. Namiki, "A new FDTD algorithm based on alternating-direction implicit method," IEEE Trans. Microw. Theory Tech., vol. 47, no. 10, pp. 2003-2007, Oct. 1999.

[6] F. H. Zheng, Z. Z. Chen, and J. Z. Zhang, "A finite-difference time-domain method without the Courant stability conditions," IEEE Microw. Guided Wave Lett., vol. 9, no. 11, pp. 441-443, Nov. 1999.

[7] B. Huang, G. Wang, Y. Jiang, and W. Wang, "A hybrid implicit-explicit FDTD scheme with weakly conditional stability," Microw. Opt. Technol. Lett., vol. 39, no. 2, pp. 97-101, Oct. 2003.

[8] J. Chen and J. Wang, "A novel body-of-revolution finite-difference time-domain method with weakly conditional stability," IEEE Microw. Wireless Compon. Lett., vol. 18, no. 6, pp. 377-379, Jun. 2008.

[9] J. Shibayama, M. Muraki, J. Yamauchi, and H. Nakano, "Efficient implicit FDTD algorithm based on locally one-dimensional scheme," Electron. Lett., vol. 41, no. 19, pp. 1046-1047, Sep. 2005.

[10] V. E. do Nascimento, J. A. Cuminato, F. L. Teixeira, and B.-H. V. Borges, "Unconditionally stable finite-difference time-domain method based on the locally-one-dimensional technique," in Proc. 22nd Symp. Brasileiro Telecomun., Campinas, Brazil, Sep. 2005, pp. 288-291.

[11] J. Shibayama, M. Muraki, R. Takahashi, J. Yamauchi, and H. Nakano, "Performance evaluation of several implicit FDTD methods for optical waveguide analyses," J. Lightw. Technol., vol. 24, no. 6, pp. 2465-2472, Jun. 2006

[12] W. Fu and E. L. Tan, "Development of split-step FDTD method with higher order spatial accuracy," Electron. Lett., vol. 40, no. 20, pp. 1252-1254, Sep. 2004.

[13] K.-Y. Jung and F. L. Teixeira, "An iterative unconditionally stable LOD-FDTD method," IEEE Microw. Wireless Compon. Lett., vol. 18, no. 2, pp. 76-78, Feb. 2008.

[14] E. L. Tan, "Unconditionally stable LOD-FDTD method for 3-D Maxwell's equations," IEEE Microw. Wireless Compon. Lett., vol. 17, no. 2, pp. 85-87, Feb. 2007.

[15] I. Ahmed, E. K. Chua, and E. P. Li, "The stability analysis of the three-dimensional LOD-FDTD method," in Proc. Asia-Pacific Symp. Electromagn. Compat., Singapore, May 2008, p. TU-CEM-1-5.

[16] Q. Liu, Z. D. Chen, and W. Yin, "An efficient unconditionally stable three-dimensional LOD-FDTD method," in Proc. Int. Microw. Symp., Atlanta, GA, Jun. 2008, p. TU3C-01.

[17] C. Yuan and Z. Chen, "A three-dimensional unconditionally stable ADI-FDTD method in the cylindrical coordinate system," IEEE Trans. Microw. Theory Tech., vol. 50, no. 10, pp. 2401-2405, Oct. 2002.

[18] C. A. Balanis, Advanced Engineering Electromagnetics. New York: Wiley, 1989, pp. 492-499.

[19] D. M. Pozar, Microwave Engineering. Boston, MA: Addison-Wesley, 1990, pp. 318-320.

[20] F. L. Teixeira and W. C. Chew, "PML-FDTD in cylindrical and spherical grids," IEEE Microw. Guided Wave Lett., vol. 7, no. 9, pp. 285-287, Sep. 1997.

[21] H.-L. Chen and B. Chen, "Anisotropic-medium PML for ADI-BORFDTD method," IEEE Microw. Wireless Compon. Lett., vol. 18, no. 4, pp. 221-223, Apr. 2008.

[22] V. E. do Nascimento, B.-H. V. Borges, and F. L. Teixeira, "Split-field PML implementations for the unconditionally stable LOD-FDTD method," IEEE Microw. Wireless Compon. Lett., vol. 16, no. 7, pp. 398-400, Jul. 2006.

[23] O. Ramadan, "Unsplit field implicit PML algorithm for complex envelope dispersive LOD-FDTD simulations," Electron. Lett., vol. 43, no. 5, pp. 267-268, Mar. 2007.

[24] I. Ahmed, E. Li, and K. Krohne, "Convolutional perfectly matched layer for an unconditionally stable LOD-FDTD method," IEEE Microw. Wireless Compon. Lett., vol. 17, no. 12, pp. 816-818, Dec. 2007.

[25] E. L. Tan, "Fundamental schemes for efficient unconditionally stable implicit finite-difference time-domain methods," IEEE Trans. Antennas Propagat., vol. 56, no. 1, pp. 170-177, Jan. 2008. 